\title{
The Continuous Newton-Raphson Method Can Look Ahead
}

\author{
Raphael Hauser* and Jelena Nedić ${ }^{\dagger}$ \\ Oxford University Computing Laboratory, Wolfson Building, Parks Road, \\ Oxford, OX1 3QD, England,UK; \{hauser, jelena\}@comlab.ox.ac.uk \\ http://web.comlab.ox.ac.uk/oucl/people/\{raphael.hauser.html; jelena.nedic.html\}
}

\begin{abstract}
This paper is about an intriguing property of the continuous Newton-Raphson method for the minimization of a continuous objective function $f$ : if $x$ is a point in the domain of attraction of a strict local minimizer $x^{*}$ then the flux line of the Newton-Raphson flow that starts in $x$ approaches $x^{*}$ from a direction that depends only on the behavior of $f$ in arbitrarily small neighborhoods around $x$ and $x^{*}$. In fact, if $\hat{f}$ is a sufficiently benign perturbation of $f$ on an open region $D$ not containing $x$, then the two flux lines through $x$ defined by the Newton-Raphson vector fields that correspond to $f$ and $\hat{f}$ differ from one another only within $D$.
\end{abstract}

Key words: Continuous Newton method, Newton-Raphson method, Newton flow, qualitative analysis.

AMS subject classification: $65 \mathrm{~K} 10,65 \mathrm{H} 20,58 \mathrm{C} 15$.

\section{Introduction}

The continuous Newton method for the solution of nonlinear systems of equations arises from Newton's method when damping with infinitesimal step sizes is applied. To numerical analysts this method is of theoretical interest because the topological behavior of the continuous dynamical system corresponding to a Newton vector field is better understood than the discrete

*Supported by EPSRC grant No GR/S34472

†'Supported by the Clarendon Fund, Oxford University Press and ORS Award, Universities UK 
dynamical systems arising from Newton's method with finite damping, see for example the papers of Smale [9], Branin [4], Janovsky-Seige [5], JongenJonker-Twilt [6] and Neuberger [7]. Such results proved to be useful in the analysis of solutions to systems of PDEs using Sobolev gradient methods where an insufficient number of boundary conditions may not directly guarantee the existence of a unique solution, see for example Ball [2] and Neuberger [8]. Anstreicher [1] investigated the continuous Newton-Raphson method in the context of barrier minimization in linear programming. This is the same as the continuous Newton method applied to the zero-finding problem defined by the barrier gradient field.

Applied to the problem of minimizing an arbitrary nonlinear function $f: \mathbb{R}^{n} \rightarrow \mathbb{R}$, the Newton-Raphson flow equations are defined by

$$
\frac{\partial}{\partial t} \varphi\left(x_{0}, t\right)=-H\left(\varphi\left(x_{0}, t\right)\right)^{-1} \nabla f\left(\varphi\left(x_{0}, t\right)\right), \quad \varphi\left(x_{0}, 0\right)=x_{0},
$$

where

$$
\begin{aligned}
\nabla f(y) & :=\left.D_{x} f(x)^{T}\right|_{x=y} \in \mathbb{R}^{n} \\
H(y) & :=\left.D_{x}^{2} f(x)\right|_{x=y} \in \mathbb{R}^{n \times n}
\end{aligned}
$$

The continuous Newton-Raphson method then consists of choosing a starting point $x_{0}$ and following the flux line $t \mapsto \varphi\left(x_{0}, t\right)$, obtained by integrating the ODE (1), to a stationary point $x^{*}=\lim _{t \rightarrow \infty} \varphi\left(x_{0}, t\right)$ of $\nabla f$.

Janovsky and Seige [5] showed that, subject to certain regularity conditions, the topological behavior of the Newton flow associated with the continuous Newton method for solving systems of equations is invariant under small perturbations of the system. In this paper we consider a more restrictive setting and prove a similar but stronger property: we consider the continuous Newton-Raphson method to minimize $f$ applied at a point $x_{0}$ in the domain of attraction of a strict local minimizer $x^{*}$. Under these conditions, if $\hat{f}$ is a "benign" perturbation of $f$ in an open domain $\mathcal{D}_{P}$ that excludes a small neighborhood of $x_{0}$, and if $\hat{\varphi}\left(x_{0}, t\right)$ denotes the flux line flowing through $x_{0}$ under the perturbed dynamical system

$$
\frac{\partial}{\partial t} \hat{\varphi}\left(x_{0}, t\right)=-\hat{H}\left(\hat{\varphi}\left(x_{0}, t\right)\right)^{-1} \nabla \hat{f}\left(\hat{\varphi}\left(x_{0}, t\right)\right), \quad \hat{\varphi}\left(x_{0}, 0\right)=x_{0},
$$


with $\nabla \hat{f}=D_{x} \hat{f}$ and $\hat{H}=D_{x}^{2} \hat{f}$, then $\varphi\left(x_{0}, t\right)$ and $\hat{\varphi}\left(x_{0}, t\right)$ differ only for values of $t$ for which $\varphi\left(x_{0}, t\right), \hat{\varphi}\left(x_{0}, t\right) \in \mathcal{D}_{P}$. In other words, the dynamics of $\varphi$ and $\hat{\varphi}$ are not only topologically equivalent but the flux lines actually coincide outside of the domain of perturbation, see Figure 1.

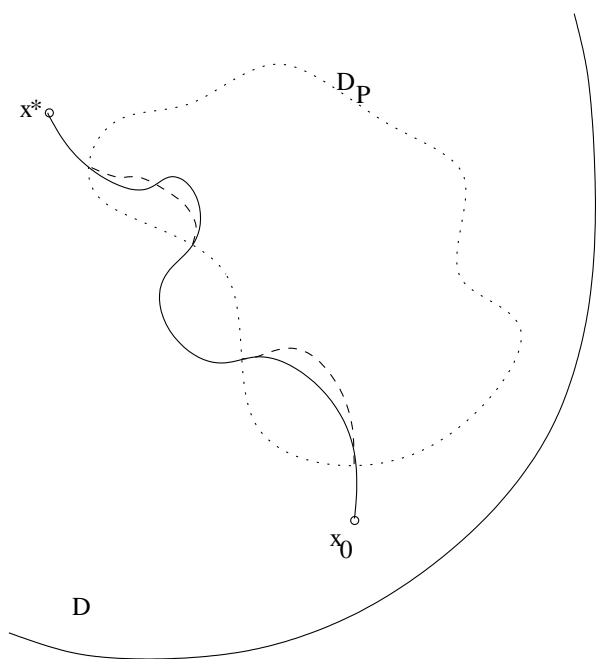

Figure 1: Flux lines of the original function $f$ (solid line) and of the function $\hat{f}$ which is perturbed in the dotted region $\mathcal{D}_{P}$ (dashed line).

This property is intriguing, because it seems counterintuitive: $\varphi\left(x_{0}, t\right)$ and $\hat{\varphi}\left(x_{0}, t\right)$ clearly must coincide for values of $t$ that occur before first entering $\mathcal{D}_{P}$, because the equations (1) and (2) coincide on this range. However, one might expect that $\hat{\varphi}$ starts to differ from $\varphi$ inside $\mathcal{D}_{P}$ and leads to an exit point $\hat{y}=\hat{\varphi}\left(x_{0}, \hat{\tau}\right) \in \partial \mathcal{D}_{P}$ from $\mathcal{D}_{P}$ which is different from the point $y=\varphi\left(x_{0}, \tau\right) \in \partial \mathcal{D}_{P}$ where $\varphi$ exits. If that was the case, then the remaining parts of the flux lines

$$
\begin{array}{ll}
\varphi\left(x_{0}, t\right)=\varphi(y, t-\tau), & (t>\tau), \\
\hat{\varphi}\left(x_{0}, t\right)=\hat{\varphi}(\hat{y}, t-\hat{\tau}), & (t>\hat{\tau})
\end{array}
$$

would differ from one another.

This intuitive picture is wrong, because a simple lemma shows that the normalized gradient

$$
\mathfrak{n}(\nabla f(y)):=\frac{\nabla f(y)}{\|\nabla f(y)\|}
$$


is an invariant for points $y$ that lie along the flux line $\varphi\left(x_{0}, t\right)$, see Lemma 2.1. The same of course holds true for $\mathfrak{n}(\nabla \hat{f})$. This mechanism forces the exit points $x$ and $\hat{x}$ and the exit times $\tau$ and $\hat{\tau}$ to coincide, which in turn implies that $\varphi$ and $\hat{\varphi}$ will perfectly coincide after exiting $\mathcal{D}_{P}$, until they next enter the domain of perturbation.

If $\mathcal{D}_{P}$ furthermore excludes a small neighborhood of $x^{*}$, then $\varphi\left(x_{0}, t\right)$ and $\hat{\varphi}\left(x_{0}, t\right)$ coincide for all $t$ large enough. Thus, the continuous NewtonRaphson method "knows" how it will approach $x^{*}$ solely on the basis of information about $f$ taken from neighborhoods around $x_{0}$ and $x^{*}$; the function values of $f$ at points that are positioned along the way have no bearing on this. For example, the limiting direction at which $\varphi\left(x_{0}, t\right)$ approaches $x^{*}$ is given by

$$
\lim _{t \rightarrow+\infty} \mathrm{e}^{t} \frac{\partial}{\partial t} \varphi\left(x_{0}, t\right)=-H\left(x^{*}\right)^{-1} \nabla f\left(x_{0}\right)
$$

see Theorem 3.1 below. Thus, in this specific sense the continuous NewtonRaphson method can "look ahead": it knows where it is going and can only be distracted but not ultimately deviated from its predetermined path by local perturbations.

For the purposes of our analysis we will assume throughout that $x^{*}$ is a point such that $\nabla f\left(x^{*}\right)=0$ and the Hessian at $x^{*}$ is positive definite $\left(H\left(x^{*}\right) \succ 0\right)$ implying that $x^{*}$ is a strict local minimizer of $f$, and that $f \in \mathcal{C}^{3}\left(\mathcal{D}_{3}\right)$ in some open neighborhood $\mathcal{D}_{3}$ of $x^{*}$. The domain of attraction of $x^{*}$ is the set of points $x \in \mathbb{R}$ such that $\lim _{t \rightarrow \infty} \varphi(x, t)=x^{*}$. It is well known that the assumption $H\left(x^{*}\right) \succ 0$ implies that the domain of attraction of $x^{*}$ contains an open neighborhood $\mathcal{D}_{a}$ of $x^{*}$ such that $H(x) \succ 0$ for all $x \in \mathcal{D}_{a}$. We will use the notation int $A, \partial A$ and $c l A$ for the interior, boundary and closure of a set $A$ respectively. Additionally, for convex set $A$ we will use $\operatorname{ext} A$ to denote the set of extreme points of $A$, that is to say the set of all points $x \in A$ such that $A \backslash\{x\}$ is convex. 


\section{Characterization of flux lines by an invari- ance property}

A simple but powerful property of the Newton-Raphson flow is that $\mathfrak{n}(\nabla f)$ is invariant along flux lines. This claim, proved in the next Lemma, has the interesting consequences that we will formalize in the main theorems.

Lemma 2.1. The direction of the gradient of the function $f$ is constant along flux lines defined by its Newton-Raphson vector field.

Proof. Let $\varphi\left(x_{0}, t\right)$ denote the flux line of the Newton-Raphson vector field defined by $f$ passing through $x_{0}$. Then

$$
\begin{aligned}
\left.\frac{\partial}{\partial t} \nabla f\left(\varphi\left(x_{0}, t\right)\right)\right) & =H\left(\varphi\left(x_{0}, t\right)\right) \frac{\partial}{\partial t} \varphi\left(x_{0}, t\right) \\
& \left.=H\left(\varphi\left(x_{0}, t\right)\right)\left(-H\left(\varphi\left(x_{0}, t\right)\right)^{-1}\right) \nabla f\left(\varphi\left(x_{0}, t\right)\right)\right) \\
& =-\nabla f\left(\varphi\left(x_{0}, t\right)\right) .
\end{aligned}
$$

Therefore,

$$
\left.\nabla f\left(\varphi\left(x_{0}, t\right)\right)=\mathrm{e}^{-t} \nabla f\left(\varphi\left(x_{0}, 0\right)\right)\right)
$$

and $\left\|\nabla f\left(\varphi\left(x_{0}, t\right)\right)\right\|=\mathrm{e}^{-t}\left\|\nabla f\left(\varphi\left(x_{0}, 0\right)\right)\right\|$, that is,

$$
\mathfrak{n}\left(\nabla f\left(\varphi\left(x_{0}, t\right)\right)\right)=\mathfrak{n}\left(\nabla f\left(\varphi\left(x_{0}, 0\right)\right)\right),
$$

which proves the claim.

\section{Limiting behavior of flux lines}

As a direct consequence of Lemma 2.1 we now obtain the following result:

Theorem 3.1. Let $x_{0} \in \mathcal{D}$. Then the limiting direction of the flux line $\varphi\left(x_{0}, t\right)$ is the normalization of the vector

$$
\lim _{t \rightarrow+\infty} \mathrm{e}^{t} \frac{\partial}{\partial t} \varphi\left(x_{0}, t\right)=-H\left(x^{*}\right)^{-1} \nabla f\left(x_{0}\right) .
$$


Proof. Using equation (3) we obtain

$$
\left.\lim _{t \rightarrow+\infty} e^{t} \frac{\partial}{\partial t} \varphi\left(x_{0}, t\right)=-\lim _{t \rightarrow+\infty} H\left(\varphi\left(x_{0}, t\right)\right)^{-1} \nabla f\left(x_{0}\right)\right)=-H\left(x^{*}\right)^{-1} \nabla f\left(x_{0}\right) .
$$

where the last equality follows from the fact that flux line $\varphi\left(x_{0}, t\right)$ converges to $x^{*}$ as $t \rightarrow \infty$ and assumption that Hessian is continuous function.

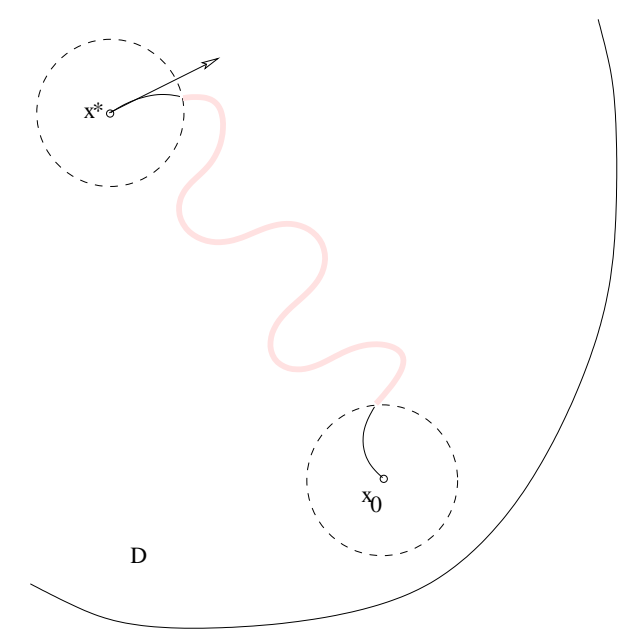

Figure 2: The limiting direction of the flux line through $x_{0}$ depends only on information in small neighborhoods of $x_{0}$ and $x^{*}$

Theorem 3.1 shows that the direction at which the flux line $\varphi\left(x_{0}, t\right)$ approaches $x^{*}$ depends only on the gradient of $f$ at $x_{0}$ and the Hessian at $x^{*}$. In other words, knowing only the behavior of the function $f$ in a small neighborhood around $x_{0}$ and $x^{*}$ one can determine the limiting direction of a flux line. Since the limiting direction does not depend on the value of $f$ at intermediate points along the flux line $\varphi\left(x_{0}, t\right)$, does this mean that if we perturb $f$ in a region that lies in its path, the behavior of $\varphi\left(x_{0}, t\right)$ around $x^{*}$ will be the same? If the perturbation is sufficiently benign the answer is yes. As a matter of fact, a much stronger claim is true: the flux lines of $f$ and its perturbation differ only in the region of perturbation! This is the subject of the next section. 


\section{Change of flux lines under perturbations of function $f$}

In this section we will prove the main theorem of this paper which we described in the introduction as "counterintuitive". Thinking back of Lemma 2.1 and its use in the proof of Theorem 3.1, the result we claim here is now more plausible: the normalized gradient of the $f$ along $\varphi\left(x_{0}, t\right)$ takes the constant value $\mathfrak{n}\left(\nabla f\left(x_{0}\right)\right)$. Moreover, under the regularity assumption we have made the set of points where $\mathfrak{n}(\nabla f)=\mathfrak{n}\left(\nabla f\left(x_{0}\right)\right)$ is one-dimensional. Since $f$ and its perturbation $\hat{f}$ are identical in the unperturbed region, the loci of the points on the flux lines $\varphi\left(x_{0}, t\right)$ and $\hat{\varphi}\left(x_{0}, t\right)$ have to lie in the same one-dimensional set, forcing them to coincide.

Before formalizing and proving this result, we need the following definitions and auxiliary results.

Definition 4.1. Let $C \subset \mathbb{R}^{n}$ and $x \in \partial C$. A supporting hyperplane of $C$ at $x$ is $S_{C}(x)=\left\{y \in \mathbb{R}^{n}:\langle a ; y-x\rangle=0\right\}$ where $0 \neq a \in \mathbb{R}^{n}$ is a vector such that for all $y \in C,\langle a ; y-x\rangle \leq 0$.

In other words, a supporting hyperplane of a set $C$ at $x$ is a hyperplane containing $x$ such that the whole set $C$ lies in one closed halfspace defined by the hyperplane. It is well known (see e.g. [3]) that if $C$ is convex, then a supporting hyperplane exists for every $x \in \partial C$.

Definition 4.2. Let $C \subset \mathbb{R}^{n}$ be a convex set and $x \in C$.

The normal cone of $C$ at $x$ is $N_{C}(x)=\left\{d \in \mathbb{R}^{n}:\langle d ; y-x\rangle \leq 0, \forall y \in C\right\}$. The tangent cone of $C$ at $x$ is $T_{C}(x)=\left\{\alpha(c-x): \alpha \in \mathbb{R}_{+}, c \in C\right\}$.

Definition 4.3. Let $A \subset \mathbb{R}^{n}$. The polar cone of $A$ is

$$
A^{-}=\left\{y \in \mathbb{R}^{N}:\langle y ; x\rangle \leq 0, \forall x \in A\right\} .
$$

It is not difficult to prove that $N_{C}(x)=T_{C}(x)^{-}$and $T_{C}(x)=N_{C}(x)^{-}$, see e.g. [3].

Using the notions defined above, we can now show that for a function with a strictly convex level set $L_{k}$, that is, $\partial L_{k}=\operatorname{ext}\left(L_{k}\right)$, there exists a unique point in $\partial L_{k}$ where $\nabla f$ has a given direction. 
Lemma 4.4. Let $f \in \mathcal{C}^{3}$ be a function with positive definite Hessian $H \succ$ 0 for every $x$ in some neighborhood $\mathcal{D}$ of a strict local minimizer $x^{*}$, and let $g$ be a given unit vector. Then for any fixed level $k>f\left(x^{*}\right)$ such that $L_{k}^{f}:=\{x: f(x) \leq k\} \subseteq \mathcal{D}$ there exists a unique point $x_{k} \in \partial L_{k}$ such that $\mathfrak{n}\left(\nabla f\left(x_{k}\right)\right)=g$.

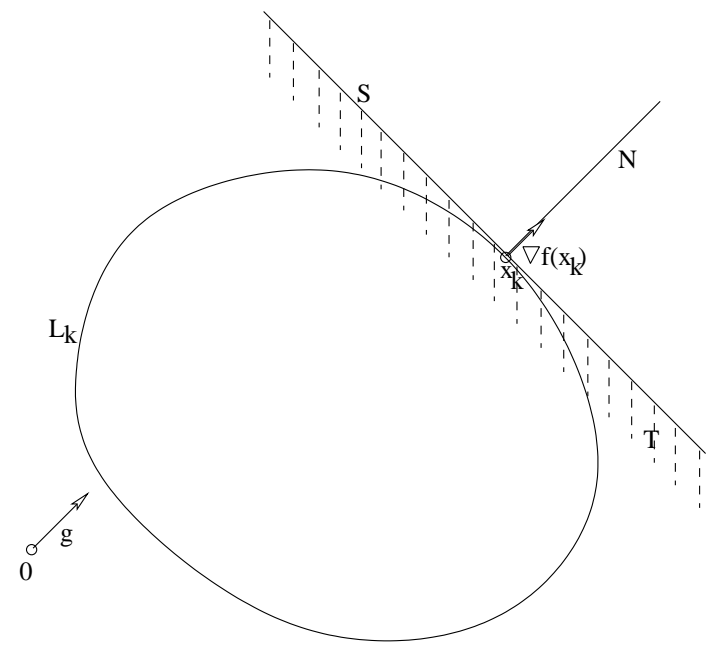

Figure 3: Supporting hyperplane $S$ defined by the vector $g$, normal cone $N$ and tangent cone $T$ of level set $L_{k}$ at $x_{k}$

Proof. It is easy to prove that the condition $H(x) \succ 0$ on $\mathcal{D}$ implies that all level sets $L_{k}^{f}=\{x: f(x) \leq k\}$ such that $L_{k} \subseteq \mathcal{D}$ are strictly convex, that is to say, $\partial L_{k}^{f}=e x t L_{k}^{f}$. Existence: Let us first find a point $x_{k} \in \partial L_{k}^{f}$ where $g$ defines a supporting hyperplane. Since we want $\left\langle g ; y-x_{k}\right\rangle=\langle g ; y\rangle-\left\langle g ; x_{k}\right\rangle \leq$ 0 to hold for every $y \in L_{k}^{f}$, let us choose $x_{k} \in \operatorname{argmax}\left\{\langle g ; x\rangle: x \in L_{k}^{f}\right\}$. We will prove that $\mathfrak{n}\left(\nabla f\left(x_{k}\right)\right)=g$. It is easy to see that $g \in N_{L_{k}^{f}}\left(x_{k}\right)$ and that $\nabla f\left(x_{k}\right) \neq 0$. By the implicit function theorem, $\partial L_{k}^{f}$ is a $\mathcal{C}^{1}$ manifold and hence $T_{\partial L_{k}^{f}}\left(x_{k}\right)$ is well defined and of dimension $n-1$. Therefore $T_{L_{k}^{f}}\left(x_{k}\right)$ is a linear halfspace and $N_{L_{k}^{f}}\left(x_{k}\right)=T_{L_{k}^{f}}\left(x_{k}\right)^{-}$is a ray (a 1-dimensional pointed cone). Since $\nabla f\left(x_{k}\right) \in N_{L_{k}^{f}}\left(x_{k}\right)$, this shows that $\mathfrak{n}\left(\nabla f\left(x_{k}\right)\right)=g$. Uniqueness: Let us now assume that there exist two points $x_{k}$ and $y_{k}$ satisfying the claim. Then, both the supporting hyperplanes for $x_{k}$ and $y_{k}$ are defined by $g$. The definition of $S_{L_{k}^{f}}\left(x_{k}\right)$ implies that $\left\langle g ; y_{k}-x_{k}\right\rangle \leq 0$, while $\left\langle g ; x_{k}-y_{k}\right\rangle \leq 0$ follows 
from the definition of $S_{L_{k}^{f}}\left(y_{k}\right)$. Hence $\left\langle g ; x_{k}-y_{k}\right\rangle=0$ and $y_{k} \in S_{L_{k}^{f}}\left(x_{k}\right)$. But this implies that the entire line segment defined by $x_{k}$ and $y_{k}$ belongs to $\partial L_{k}^{f}$, so for every $\lambda \in(0,1)$ we have that $\lambda x_{k}+(1-\lambda) y_{k} \in \partial L_{k}^{f} \backslash \operatorname{ext} L_{k}^{f}$ which contradicts the assumption that $L_{k}^{f}$ is strictly convex.

Hereafter we will say that the function $\hat{f}$ is a regular perturbation of $f \in \mathcal{C}^{3}$ with local minimizer $x^{*}$ if the following conditions are satisfied:

(i) $\hat{f} \in \mathcal{C}^{3}$ and $H_{\hat{f}} \succ 0, \forall x \in \mathcal{D}$,

(ii) $f(x)-\hat{f}(x) \neq 0 \Rightarrow x \in \mathcal{D}_{P} \subset \mathcal{D}$ where $\mathcal{D}_{P}$ is an open set.

Note that the condition (i) insures that the Newton-Raphson vector field is well defined and guarantees no bifurcation of flux lines. Moreover, we will use the notation

$$
\Gamma_{\varphi}\left(x_{0}\right)=\left\{x: x=\varphi\left(x_{0}, t\right) \text { for some } t \geq 0\right\},
$$

to denote the flux line $\varphi\left(x_{0}, t\right)$ as a set of points and we write $A \triangle B$ for the symmetric difference between two sets $A$ and $B$.

All the tools are now available to formalize and prove the main result of this paper: the Newton-Raphson-flux lines defined by a regular perturbation $\hat{f}$ of $f$ differ from the Newton-Raphson-flux lines defined by $f$ only in the region of perturbation $\mathcal{D}_{P}$.

Theorem 4.5. Let $f$ be a $\mathcal{C}^{3}$ function with $H_{f} \succ 0$ for all $x \in \mathcal{D}$ and let $x^{*} \in \mathcal{D}$ be a strict local minimizer of $f$. Let $\hat{f}$ be a regular perturbation of $f$, and let $\varphi\left(x_{0}, t\right)$ and $\hat{\varphi}\left(x_{0}, t\right)$ be the Newton-Raphson-flows defined by $f$ and $\hat{f}$. Let $x_{0}$ be a point in $\mathcal{D} \backslash \operatorname{cl}\left(\mathcal{D}_{P}\right)$ such that $L_{f\left(x_{0}\right)}^{f} \subset \mathcal{D}$ and $L_{\hat{f}\left(x_{0}\right)}^{\hat{f}} \subset \mathcal{D}$. Then

$$
\Gamma_{\varphi}\left(x_{0}\right) \triangle \Gamma_{\hat{\varphi}}\left(x_{0}\right) \subset \mathcal{D}_{P}
$$

and $\varphi\left(x_{0}, t\right)=\hat{\varphi}\left(x_{0}, t\right)$ for all $t \in \mathbb{R}$ such that $\varphi\left(x_{0}, t\right) \notin \mathcal{D}_{P}$.

Proof. Let $g=\mathfrak{n}\left(\nabla f\left(x_{0}\right)\right)=\mathfrak{n}\left(\nabla \hat{f}\left(x_{0}\right)\right)$ where the latter equality holds because $f=\hat{f}$ in a neighborhood of $x_{0}$. We claim that

$$
\begin{gathered}
\Gamma_{\varphi}\left(x_{0}\right)=\left\{x \in \mathcal{D}: \mathfrak{n}(\nabla f(x))=g, f(x) \leq f\left(x_{0}\right)\right\}=: \Upsilon_{g}\left(x_{0}\right) \\
\Gamma_{\hat{\varphi}}\left(x_{0}\right)=\left\{x \in \mathcal{D}: \mathfrak{n}(\nabla \hat{f}(x))=g, \hat{f}(x) \leq \hat{f}\left(x_{0}\right)\right\}=: \hat{\Upsilon}_{g}\left(x_{0}\right)
\end{gathered}
$$


Since $H \succ 0$ and $H_{f} \succ 0$, level sets $L_{k}^{f}$ and $L_{k}^{\hat{f}}$ are strictly convex in $\mathcal{D}$ for every $k \leq f\left(x_{0}\right)$. Therefore $\Gamma_{\varphi}\left(x_{0}\right) \subseteq \Upsilon_{g}\left(x_{0}\right)$ by Lemma 2.1. On the other hand, assume that there exist a point $y \in \Upsilon_{g}\left(x_{0}\right) \backslash \Gamma_{\varphi}\left(x_{0}\right)$. We know that $x_{0} \in \Gamma_{\varphi}\left(x_{0}\right)$ so $y \neq x_{0}$. Since $f$ is continuous and strictly decreasing along $\Gamma_{\varphi}\left(x_{0}\right)$ there exists a point $x_{1} \in \Gamma_{\varphi}\left(x_{0}\right)$ such that $f\left(x_{1}\right)=f(y)<f\left(x_{0}\right)$ and $\mathfrak{n}\left(\nabla f\left(x_{1}\right)\right)=\mathfrak{n}\left(\nabla f\left(x_{0}\right)\right)=\mathfrak{n}(\nabla f(y))$, where the first equality follows from the Lemma 2.1 and the second one from the fact that $y \in \Upsilon_{g}\left(x_{0}\right)$. This implies that there are two distinct points in the level set $L_{f(y)}^{f}$ with the same gradient direction, in contradiction to Lemma 4.4. Therefore $\Gamma_{\varphi}\left(x_{0}\right)=\Upsilon_{g}\left(x_{0}\right)$. The result for the function $\hat{f}$ can be derived in a similar fashion. Now, since $f(x)=\hat{f}(x)$ for $x \in \mathcal{D} \backslash \mathcal{D}_{P}$ and in particular $f\left(x_{0}\right)=\hat{f}\left(x_{0}\right)$, it is easy to see that $\Upsilon_{g}\left(x_{0}\right)$ and $\hat{\Upsilon}_{g}\left(x_{0}\right)$ are the same in the unperturbed region, i.e. $\Upsilon_{g}\left(x_{0}\right) \cap\left(\mathcal{D} \backslash \mathcal{D}_{P}\right)=\hat{\Upsilon}_{g}\left(x_{0}\right) \cap\left(\mathcal{D} \backslash \mathcal{D}_{P}\right)$. In other words, $\Gamma_{\varphi}\left(x_{0}\right) \cap\left(\mathcal{D} \backslash \mathcal{D}_{P}\right)=$ $\Gamma_{\hat{\varphi}}\left(x_{0}\right) \cap\left(\mathcal{D} \backslash \mathcal{D}_{P}\right)$, showing the first claim. The last claim now follows from Equation (3).

\section{$5 \quad$ Numerical examples}

\subsection{A two-dimensional example}

Let us consider the quadratic function $f(x)=c+b^{T} x+\frac{1}{2} x^{T} B x$ for $c=$ $2, b=(1,2)^{T}$ and $B=\left(\begin{array}{ll}2 & 1 \\ 1 & 4\end{array}\right)$ with global minimizer $x^{*}=(6 / 7,-5 / 7)^{T}$ and perturb it in an annulus around $x^{*}$ with inner and outer radii $r_{a}$ and $r_{b}$ respectively. Let us choose a perturbation term given by a polynomial of degree 8 with 4 -tuple zeros $r_{a}$ and $r_{b}: \hat{f}(x)=f(x)+p\left(\left\|x-x^{*}\right\|\right)$, where

$$
p(s)=\left\{\begin{array}{cl}
\varepsilon\left(s-r_{a}\right)^{4}\left(s-r_{b}\right)^{4}, & \text { for } r_{a}<s<r_{b}, \\
0, & \text { otherwise. }
\end{array}\right.
$$

The following figures show the results for $r_{a}=1, r_{b}=3$ and $\varepsilon=1$ obtained in numerical computations performed in Matlab using the ODE integrator ode45: Figure 4 shows a few level sets of the perturbed function $\hat{f}$, the annulus where the original function was perturbed and the flux lines $\varphi$ and $\hat{\varphi}$ respectively corresponding to $f$ (solid lines) and $\hat{f}$ (dashed curves). It can be clearly seen that the flux lines differ only in the region of perturbation, as proven in Theorem 4.5. In fact, this example shows that the convexity 
assumptions of Theorem 4.5 are sufficient but not necessary for its claim to hold.

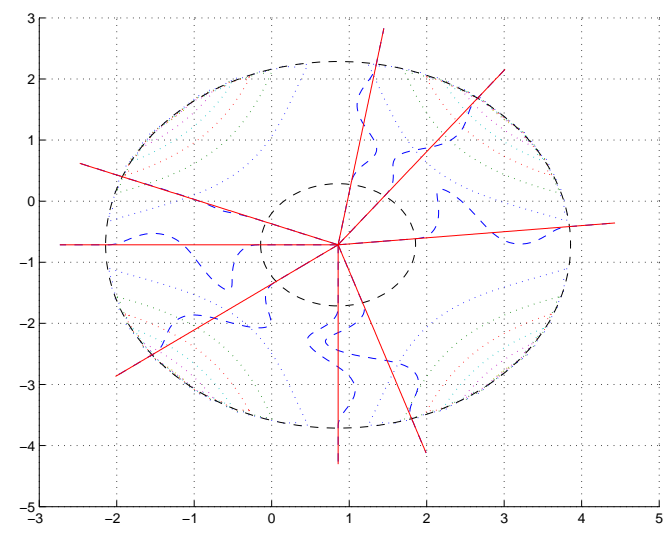

Figure 4: Perturbed region, level sets of the perturbed function, and flux lines of the original (solid line) and the perturbed (dashed curves) function.

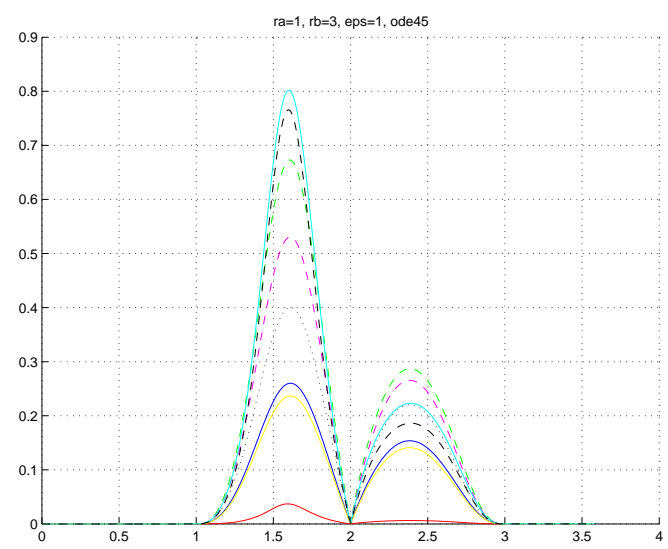

Figure 5: Norms of the difference of flux lines for $f$ and $\hat{f}$ as functions of $\left\|x-x^{*}\right\|$

Note that the "time scale" of the flux lines for $f$ and $\hat{f}$ is in general different in the perturbed region. Therefore, in order to measure the difference between points on $\Gamma_{\varphi}\left(x_{0}\right)$ and $\Gamma_{\hat{\varphi}}\left(x_{0}\right)$, we have reparametrised the flux lines in terms of the distance to $x^{*}$. Accordingly, Figure 5 shows the norm 
of the difference of flux lines shown in Figure 4 as functions of $\left\|x-x^{*}\right\|$. The flux lines differ only in the region $1<\left\|x-x^{*}\right\|<3$, confirming the claim of Theorem 4.5. Note that $p(s)$ has exactly one local extremum $s^{*}$ for $r_{a}<s<r_{b}$. Therefore, for $\bar{x}$ such that $\left\|\bar{x}-x^{*}\right\|=s^{*}$ we have $\nabla \hat{f}(\bar{x})=\nabla f(\bar{x})+\left.p^{\prime}\left(s^{*}\right) \frac{d}{d x}\left\|x-x^{*}\right\|\right|_{x=\bar{x}}=\nabla f(\bar{x})$ so recalling the proof of Theorem 4.5 we see that the perturbed and unperturbed flux lines intersect at such points, which explains the dip observed in Figure 5.

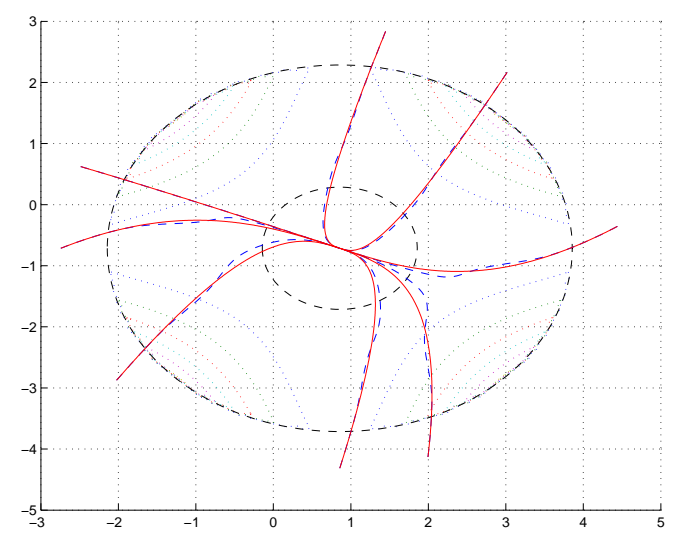

Figure 6: Flux lines of the negative gradient field for the original (solid line) and the perturbed (dashed curves) function.

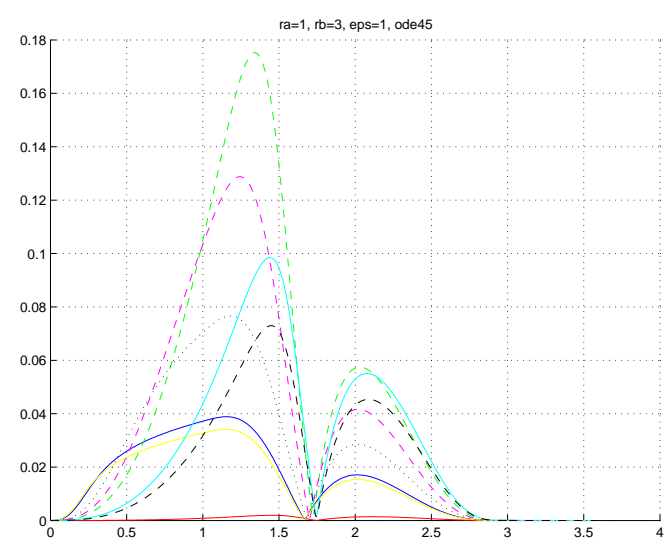

Figure 7: Norms of the difference of the negative gradient field flux lines for $f$ and $\hat{f}$ as functions of $\left\|x-x^{*}\right\|$ 
In order to emphasis the fact that this property is closely related to Newton-Raphson vector field and does not necessarily hold for all vector fields, the same tests were performed on negative gradient field and the results are shown in Figures 6 and 7 . It is clear that flux lines for the perturbed function exit the region of perturbation at the points different from exit points of the flux lines for the original function and therefore converge to the solution $x^{*}$ following different flows.

\subsection{A three-dimensional example}

The following 3-dimensional example was constructed in a similar fashion. Let us again consider the quadratic function $f(x)=c+b^{T} x+\frac{1}{2} x^{T} B x$ and its perturbation $\hat{f}=f(x)+p\left(\left\|x-x^{*}\right\|\right)$ which differs from $f$ on a sphere of inner radius $r_{a}$ and outer radius $r_{b}$ around the exact minimizer $x^{*}$. The perturbation term is given by the polynomial $p(s)$ defined in (4).

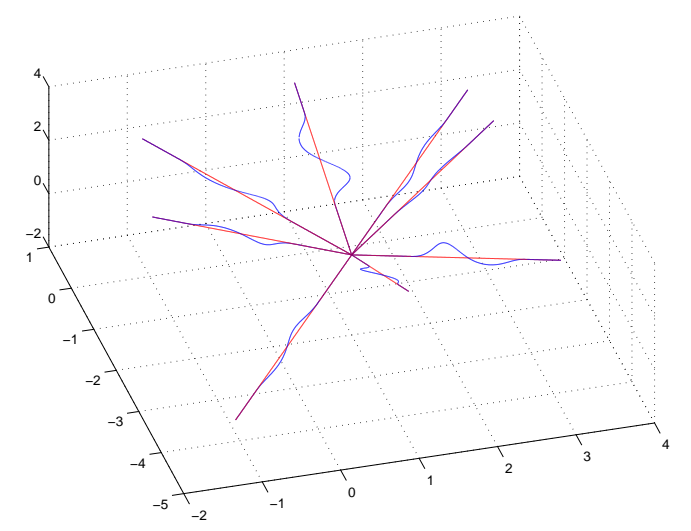

Figure 8: Flux lines of original and perturbed function in 3D case

The particular values $r_{a}=1, r_{b}=3, \varepsilon=1, c=2, b=(2,-5,3)^{T}$ and

$$
B=\left(\begin{array}{lll}
5 & 2 & 1 \\
2 & 4 & 1 \\
1 & 1 & 4
\end{array}\right)
$$

were used in the numerical experiments. In this case the exact minimizer is $x^{*}=(1,-2,1)^{T}$. 


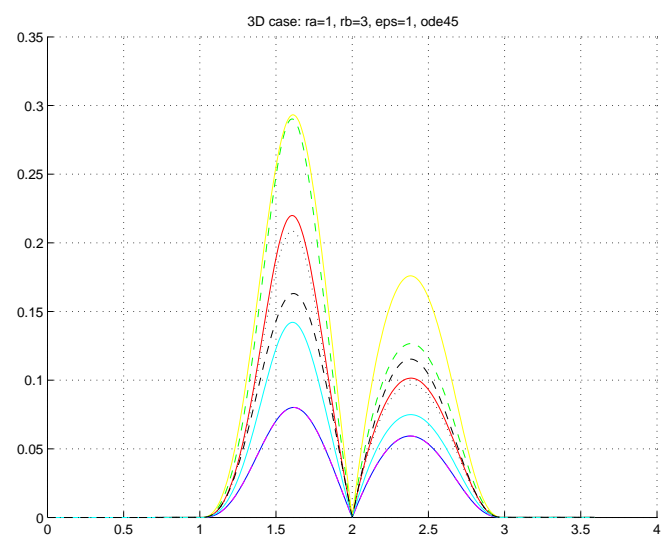

Figure 9: Norms of a difference of flux lines for $f$ and $\hat{f}$ as functions of $\left\|x-x^{*}\right\|$ in $3 \mathrm{D}$ case

Figures 8 and 9 show that the flux lines for $f$ and $\hat{f}$ again differ only in the region of perturbation.

\section{Discussion}

The main theorems of this paper highlight an interesting property of the globaly dynamics of the continuous Newton-Raphson method. For the purposes of a succinct analysis we assumed that $f$ and its perturbation $\hat{f}$ are both strictly convex functions. This assumption is merely a sufficient condition that could be relaxed in many ways. In fact, using the topological results of Janovsky and Seige [5], it is in principle possible to extend the results presented here to the case where perturbations are small and where neither $H(x)$ nor $\hat{H}(x)$ is singular anywhere along $\varphi\left(x_{0}, t\right)$ and $\hat{\varphi}\left(x_{0}, t\right)$, guaranteeing that the flux lines cannot bifurcate. Our numerical experiments confirm this where we have chosen nonconvex examples which tend to make the claim of Theorem 4.5 more clearly visible than convex examples.

\section{References}

[1] K. Anstreicher, Linear programming and the Newton-barrier flow, Mathematical Programming (Series A) 41 (1988) 367-373. 
[2] J. M. Ball, Continuity properties and global attractors of generalized semiflows and the Navier-Stokes equations, Nonlinear Sci. 7 (1997) 475502.

[3] J. M. Borwein and A. S. Lewis, Convex Analysis and Nonlinear Optimization: Theory and Examples, Springer, New York, 2000.

[4] F. H. Branin, A widely convergent method for finding multiple solutions of simultaeneous nonlinear equations, IBM J. Res. Develop. 16 (1972) $504-522$.

[5] V. Janovsky and V. Seige, Qualitative analysis of Newton's flow, SIAM J. Numer. Anal. 33 No.5 (1996) 2068-2097.

[6] H. Th. Jongen, P. Jonker and F. Twilt, The continuous desingularized Newton method for meromorphic functions, Acta Appl. Math. 13 (1988) $81-121$.

[7] J. W. Neuberger, Continuous Newton's method for polynomials, Mathematical Intelligencer, Vol. 21(3), (1999) 18-23.

[8] J. W. Neuberger, Sobolev Gradients and Differential Equations, Springer Lecture Notes in Mathematics Vol. 1670, Springer-Verlag, New York, 1997.

[9] S. Smale, A convegence process in price adjustment and global Newton methods, J. Math. Econ. 3 (1976) 107-120. 